\title{
La correction du débit en présence d'une fuite entre trachée et sonde endotrachéale
}

\author{
Eve Bijaoui Ms, P-Y Carry MD PHD, \\ A Eberhard ${ }^{\star}$ PHD, P Andrinit $\mathrm{MD}$, \\ J-P Perdrix MD, P Baconnier PHD
}

Objectifs: Présenter une méthode d'estimation de la fuite entre trachée et sonde endotrachéale chez le nouveau-né afin de compenser l'erreur sur la mesure du débit et de surveiller les caractéristiques mécaniques respiratoires par l'analyse des signaux de débit et de pression mesurés à l'origine de la sonde.

Méthodes: On suppose la résistance de fuite $(R f)$ constante sur un cycle et on mesure les caractéristiques résistives du tube endotrachéal. La méthode a été validée avec un modèle mécanique de poumon et appliquée aux enregistrements de trois nouveau-nés prématurés admis en réanimation néonatale pour des détresses respiratoires. Une méthode des moindres carrés a perrmis d'estimer, avant et après correction du débit, la pression positive de fin d'expiration (PEEP).

Résultats: Pour les simulations, l'estimation de $R f$ concorde avec la mesure directe. En clinique, l'efficacité de la correction est démontrée: le débit cornigé revient à zéro pendant la pause de fin d'inspiration. En simulation, avant correction du débit. la PEEP est sous-estimée de 10 a $20 \mathrm{~cm} \mathrm{H}_{2} \mathrm{O}$. alors qu'après correction l'erreur est inférieure à $2 \mathrm{~cm} \mathrm{H}_{2} \mathrm{O}$. En clinique, la PEEP estimée passe de valeurs négatives $\left(-0,3 \pm 1,3 \mathrm{~cm} \mathrm{H}_{2} \mathrm{O}\right.$ avant correction $)$ à des valeurs positives $\left(3.6 \pm 0.7 \mathrm{~cm} \mathrm{H} \mathrm{O}_{2} \mathrm{O}\right.$ après correction) supérieures à la PEEP imposée $\left(2 \mathrm{~cm} \mathrm{H} \mathrm{H}_{2} \mathrm{O}\right)$.

Conclusions: L'efficacité de cette méthode simple a été démontrée. Elle pourrait être utilisée avec profit sur des patients adultes, la correction sur le débit n'étant pas effectuée en l'absence de fuite.

Purposes: To estimate the leak between the endotracheal tube and the trachea in newborns in order to compensate for errors in airflow measurement and to monitor mechanical variables from pressure and flow signals.

Methods: Assuming that the leak resistance $(R f)$ is constant during a respiratory cycle, the resistive properties of the endotracheal tube were evaluated. The method was validated in the intensive care unit with a mechanical test lung and assessed on recordings of three newborns during mechanical ventilation for RDS. We have used a least squares method for the estimation of positive end expiratory pressure (PEEP) on both newboms and simulated data.

Results: Direct measurements of simulated leak resistances on the mechanical lung are in agreement with our estimation of leak resistances. In newborns, the success of flow correction is evidenced on end inspiratory pauses: corrected flow drops to zero while raw data show a constant nonzero flow. On the simulated lung, the PEEP underestimation with uncorrected flow ranges from 10 to $20 \mathrm{~cm} \mathrm{H} \mathrm{O}_{2} \mathrm{O}$ while the corresponding underestimation with corrected flow is less than $2 \mathrm{~cm} \mathrm{H}_{2} \mathrm{O}$. In newborns, the flow correction shifts the estimated PEEP from negative values $\left(-0.3 \pm 1.3 \mathrm{~cm} \mathrm{H}_{2} \mathrm{O}\right.$ before correction) to positive values $\left(3.6 \pm 0.7 \mathrm{~cm} \mathrm{H}_{2} \mathrm{O}\right.$ after correction) higher than the imposed PEEP $\left(2 \mathrm{~cm} \mathrm{H}_{2} \mathrm{O}\right)$.

Conclusions: The efficiency of this simple method has been demonstrated. It could be used successfully on adult patients, as there will not be flow correction in the absence of leaks.

TIMC/IMAG, Faculté de Medecine, Universite I Fourier Grenoble I, France," LMC/IMAG, Universite J Fourier Grenoblel, Rtanimation Nitonamle, CHU Grenoble.

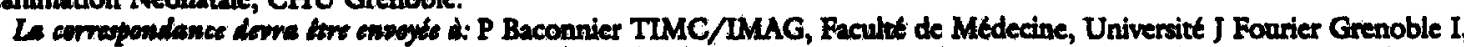
France - 38706 Le Tronche Cedex TEL: (33) 767650 47; FAX: (33) 767688 44; e-mail: pierre.baconnieraimag.fi Accepat pow publication le 26 Octobre 1996. 


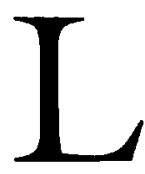

ES nouveau-nés ventilés en réanimation néonatale sont intubés avec des sondes endotrachéales sans ballonnet, d'une part, pour éviter des traumatismes de la trachée que le ballonnet peut induire et, d'autre part, parce que les sondes sans ballonnet maximisent le diamètre interne disponible. Ces sondes ne peuvent assurer l'étanchéité de la liaison sonde-trachée et on observe une fuite entre la sonde et la trachée dans la plupart des cas. ${ }^{\text {I Cette fuite }}$ présente deux inconvénients: d'une part, il est difficile d'assurer une ventilation pulmonaire correcte en présence d'une fuite, et, d'autre part, il n'est plus possible d'utiliser les signaux de débit et de pression mesurés à l'entrée de la sonde endotrachéale pour estimer les caractéristiques mécaniques du système respiratoire. ${ }^{1}$ Nous présentons une méthode de correction de ce débit basée sur l'hypothèse que la résistance de fuite $(R f)$ est constante. Une fois la fuite connue, il est possible 1) si la fuite est trop importante, de remplacer la sonde endotrachéale par une sonde de diamètre supérieur, 2) sinon, d'estimer le débit pulmonaire vrai et donc d'appliquer des méthodes maintenant classiques d'analyse des signaux débit et pression ${ }^{2}$ qui permettent de surveiller les caractéristiques mécaniques du système respiratoire. Cette méthode de calcul de $R f$ a été validée à l'aide d'un modèle mécanique de poumon et appliquée sur des enregistrements de nouveau-nés. Nous avons en particulier recherché quel était l'effet d'une telle correction sur l'estimation de la pression élastique de fin d'expiration obtenue à l'aide de la méthode des moindres carrés appliquée aux signaux débit et pression. ${ }^{2,3}$

\section{Méthodologie}

I. Théorie

1. CALCUl DE $R f$ À PARTIE DES SIGNAUX DE DÉBIT ET PRESSION

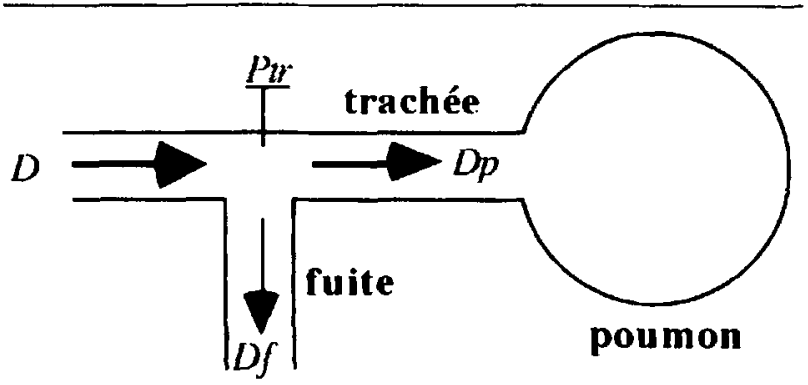

FIGURE 1 Représentation schématique du circuit respiratoire avec une fuite

Ptr: pression trachéale à la sortie de la sonde; $D$ : débit dans la sonde trachéale (gaz provenant du respirateur); $D_{p}$ : débit pulmonaire; Df: débit de fuite.
On suppose que la résistance de fuite $(R f)$ est constante sur un cycle respiratoire, c'est à dire qu'elle ne dépend pas du débit ou de la pression. En caractérisant les différents écoulements dans le circuit respiratoire par des relations pression-débit classiques, et en admettant qu'en état stationnaire, l'intégrale sur un cycle du débit des voies aériennes (débit pulmonaire) est nulle, la résistance de fuite peut être estimée cycle à cycle. Les calculs développés en annexe montrent que cette résistance s'exprime par:

$$
R f=\frac{\int P d r d t}{\int_{\text {oyde }} D \cdot d t}
$$

où Ptr est la pression trachéale, assimilée à la pression à l'extrémité de la sonde endotrachéale et $\mathrm{D}$ est le débit de gaz provenant du respirateur. Il suffit donc de déterminer la pression trachéale $\operatorname{Ptr}(t)$, le débit $D(t)$ et la pression $P(t)$ à l'entrée de la sonde étant connus. Le Tableau I regroupe les unités et les abréviations des grandeurs utilisées.

TABLEAU I : récapitulatif des différentes abréviations

\begin{tabular}{|c|c|}
\hline \multicolumn{2}{|l|}{ Pressions en $\mathrm{cm} \mathrm{H}_{2} \mathrm{O}$} \\
\hline Ptr & pression trachéale à la sortic de la sonde \\
\hline $\mathbf{P}$ & pression dans les voies aériennes \\
\hline PEEP & pression positive de fin d'expiration \\
\hline PEEP $_{\text {mes }}$ & $\begin{array}{l}\text { valcur de la pression dans le soufflet à la } f \\
\text { de l'expiration }\end{array}$ \\
\hline PEEPnC & PEEP estimée avant la correction du débi \\
\hline PEEPC & PEEP estimée après la correction du débi \\
\hline \multicolumn{2}{|l|}{ Débits en L-sec ${ }^{-1}$} \\
\hline D & $\begin{array}{l}\text { débit de gaz (provenant du respirateur) } \\
\text { dans la sonde trachéale }\end{array}$ \\
\hline Df & débit de fuite \\
\hline Dp & débit pulmonaire \\
\hline \multicolumn{2}{|c|}{ Résistances en cm H${ }_{2} \mathrm{O} \cdot \mathrm{L}^{-1} \cdot \mathrm{sec}^{-1}$} \\
\hline $\mathrm{Rf}$ & résistance de fuite \\
\hline Rf, mes & Rf mesurée directement \\
\hline Rf, calc & Rf calculée \\
\hline Rtt & $\begin{array}{l}\text { résistance du tube trachéal utilisé, } \\
\text { caractérisé par } K_{1} \text { et } K_{2}\end{array}$ \\
\hline Constantes & \\
\hline $\begin{array}{l}\mathrm{K}_{1} \mathrm{~cm} \mathrm{H} \mathrm{H}_{2} \mathrm{O} \cdot \mathrm{L}^{-1} \cdot \mathrm{sec}^{-1} \\
\mathrm{~K}_{2} \mathrm{~cm} \mathrm{H} \mathrm{H}_{2} \mathrm{O} \cdot \mathrm{L}^{-2} \cdot \mathrm{sec}^{-2}\end{array}$ & $\begin{array}{l}\text { Ces deux constantes sont telles que } \\
\text { Rtt }=K_{1}+K_{2} . \text { IDI }\end{array}$ \\
\hline
\end{tabular}

\section{Estimation de la pression à l'extrémité de la sonde} L'objectif du présent travail est de fournir une méthode de correction du débit permettant l'analyse des signaux pression et débit pour la surveillance des caractéristiques mécaniques du système respiratoire. La scule pression mesurée couramment par les respirateurs est la pression 
des voies aériennes $(P(t))$, à la sortie du ventilateur ou à l'entrée de la sonde trachéale au niveau de la pièce en Y. Il faut donc, pour mettre en ouvre notre méthode, estimer la pression trachéale à l'extrémité de la sonde. Pour cela, il suffit de connaître les propriétés résistives du circuit entre le point de mesure et l'extrémité de la sonde, en général caractérisées par l'équation de Rohrer ${ }^{2}$ :

$$
P(t)-\operatorname{Ptr}(t)=\mathrm{K}_{1} \cdot D+\mathrm{K}_{2} \cdot D|D|
$$

Une fois $\mathrm{K}_{1}$ et $\mathrm{K}_{2}$ connus, il est facile d'obtenir $\operatorname{Ptr}(t)$ à partir de $P(t)$ et $D(t)$.

\section{Protocoles expérimentaux}

l. SIMULATIONS

Afin de valider notre méthode, nous avons effectué des simulations à l'aide d'un modèle physique de poumon (Model 2600i Dual Adult TTL, Michigan Instruments)

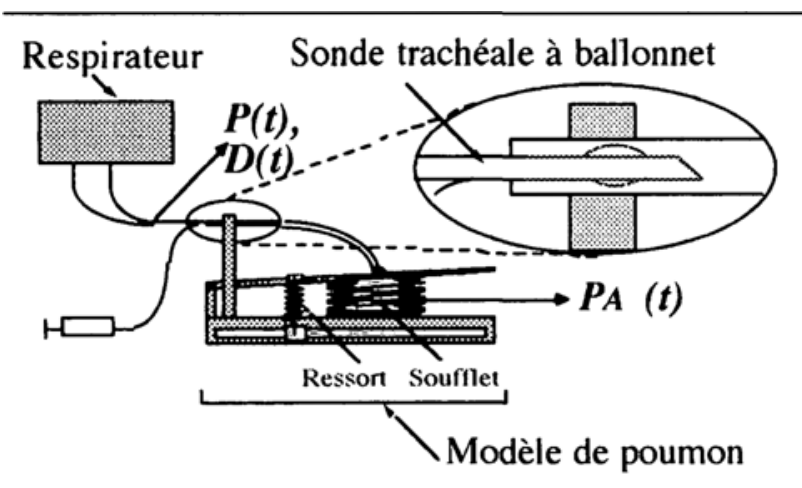

FIGURE 2 Montage expérimental des simulations de fuite Le respirateur fournit les signaux $P(t)$ et $D(t)$ à l'entrée de la sonde trachéale. La seringue permet de gonfler plus ou moins le ballonnet et de faire varier ainsi la fuite entre la sonde trachéale et lc tube de connexion du soufflet simulant la trachée. La résistance du tube reliant le soufflet à l'extéricur est considérée comme négligeable ce qui permet d'assimiler la pression dans le soufflet $(P A(t))$ à la pression à l'extrémité de la sonde. Le ressort permet de régler l'élastance du modèle de poumon.

relié à un respirateur (César, TAEMA) par une sonde trachéale à ballonnet (diamètre interne $5,5 \mathrm{~mm}$ ) insérée dans le tube reliant le soufflet à l'extérieur (Figure 2). Ce montage permet, en gonflant plus ou moins le ballonnet, de simuler une fuite variable entre sonde et trachée. Nous avons enregistré simultanément les pressions et débit (respectivement $P(t)$ et $D(t)$ ) délivrés par le respirateur et la pression dans le soufflet du modèle physique de poumon $\left(P_{A}(t)\right)$, assimilée à la pression à l'extrémité de la sonde trachéale. Les composantes de la résistance du tube trachéal à ballonnet $\left(\mathrm{K}_{\mathrm{ITB}}, \mathrm{K}_{\mathrm{2TB}}\right)$ ont été obtenues par l'étude de la relation entre la pression résis- tive $P(t)-P_{A}(t)$ et le débit $(D(t))$ lorsque le ballonnet était assez gonflé pour assurer une étanchéité parfaite. Nous n'avons pas trouvé de différence significative entre les valeurs mesurées à l'inspiration et celles mesurées à l'expiration. ${ }^{4}$ Les valeurs que nous avons utilisées sont donc des moyennes entre ces deux conditions: $\mathrm{K}_{\mathrm{ITB}}=$ $2,1 \pm 0,1 \mathrm{~cm} \mathrm{H}_{2} \mathrm{O} \cdot \mathrm{L}^{-1} \cdot \mathrm{s}$ et $\mathrm{K}_{2 \mathrm{~TB}}=27,4 \pm 0,8 \mathrm{~cm}$ $\mathrm{H}_{2} \mathrm{O} \cdot \mathrm{L}^{-2} \cdot \mathrm{sec}^{2}$. L'élastance du modèle de poumon a été fixée à $100 \mathrm{~cm} \mathrm{H} \mathrm{H}_{2} \mathrm{O} \cdot \mathrm{L}^{-1}$ afin de simuler au mieux les caractéristiques du poumon d'un nouveau-né. Le ventilateur était en mode Ventilation Contrôlée à pression constante pendant l'inspiration avec une pression positive de fin d'expiration (PEEP) imposée de $4 \mathrm{~cm} \mathrm{H}_{2} \mathrm{O}$ et un rapport des durées inspiratoire/expiratoire égal à $2 / 1$ ( 5 cycles) ou $3 / 1$ ( 7 cycles) pour obtenir des hyperinflations dynamiques significatives. La résistance de fuite a été réglée à cinq niveaux différents.

\section{Enregistrements de nouveau-nés}

Les parents des enfants étaient prévenus de l'utilisation des données recueillies à des fins de recherche. Cette étude a obtenu l'accord du comité d'éthique local. Nous avons enregistré les signaux pression et débit $(P(t)$ et $D(t))$ fournis par le respirateur chez des nouveau-nés prématurés admis dans le service de Réanimation néonatale, intubés et ventilés artificiellement (Babylog 8000, Draeger) pour des détresses respiratoires. Parmi ces patients, nous en avons sélectionné trois pour lesquels une fuite importante pouvait être mise en évidence par la différence entre les volumes courants inspiré et expiré.

Le Tableau II résume les caractéristiques de ces trois nouveau-nés prématurés (âge gestationnel compris entre 26 et 33 semaines, l'enfant $n^{\circ} 2$ a été enregistré à deux dates différentes).

Le respirateur fonctionne comme un générateur de pression: il a pour consigne, pendant la phase inspiratoire, d'augmenter progressivement la pression des voies aériennes puis de se stabiliser à une pression maximale donnée et, pendant la phase expiratoire, d'imposer une pression basse. On règle la pente de montée en pression, la pression maximale, la pression expiratoire, la durée de l'inspiration et la durée totale du cycle. Les sondes d'intubation sont des sondes trachéales pédiatriques de diamètre interne $3 \mathrm{~mm}$. Les valeurs de $\mathrm{K}_{1}$ et $\mathrm{K}_{2}$ correspondantes $\left(\mathrm{K}_{1 \mathrm{NN}}, \mathrm{K}_{2 \mathrm{NN}}\right)$ ont été obtenues en étudiant la relation pression-débit d'un tel tube trachéal isolé :

$\mathrm{K}_{\mathrm{INN}}=21,9 \pm 1,0 \mathrm{~cm} \mathrm{H} 2 \mathrm{O} \cdot \mathrm{L}^{-1} \cdot \mathrm{sec}^{-2}$ et

$\mathrm{K}_{2 \mathrm{NN}}=327,3 \pm 8,1 \mathrm{~cm} \mathrm{H} \mathrm{H}_{2} \mathrm{O} \cdot \mathrm{L}^{-2} \cdot \mathrm{sec}^{-2}$.

Aucune modification du processus de soins n'a été induite par les enregistrements. Afin de pouvoir 
TABLEAU II : caractéristiques des nouveau-nés étudiés

\begin{tabular}{cccccccccc}
\hline $\begin{array}{c}\text { Patient } \\
n^{\circ}\end{array}$ & $\begin{array}{c}\text { Age } \\
(\text { jours })\end{array}$ & $\begin{array}{c}\text { Poids } \\
(g)\end{array}$ & Pathologie & $\begin{array}{c}\text { Mode } \\
\text { ventilatoire }\end{array}$ & $\begin{array}{c}\text { FiO } \\
(\%)\end{array}$ & $\begin{array}{c}\text { PMax } \\
\left(\mathrm{cm} \mathrm{H}_{2} \mathrm{O}\right)\end{array}$ & $\begin{array}{c}\text { FR } \\
\left(\mathrm{cps} \text { min }^{-1}\right)\end{array}$ & $\begin{array}{c}\text { Rapport } \\
\text { I/E }\end{array}$ & $\begin{array}{c}\text { nb cycles } \\
\text { retentus }\end{array}$ \\
\hline 1 & 5 & 1670 & MMH & VAC & 30 & 22 & 50 & $1 / 1,5$ & 8 \\
2 & 11 & 1600 & MMH stade 4 & VACI & 35 & 20 & 40 & $2 / 1$ & 14 \\
& 18 & 1740 & MMH $\rightarrow$ DBP & VACI & 25 & 18 & 40 & $1 / 1$ & 7 \\
3 & 7 & 1610 & MMH sévère & VC & 21 & 24 & 50 & $1 / 1$ & 3 \\
\hline
\end{tabular}

VAC: ventilation assistéc contrôlée ; VACI: ventilation assistée contrôlée intermittente ; VC: ventilation contrôléc; MMH: maladie des membranes hyalines ; DPB: dysplasie broncho-pulmonaire; $\mathrm{FiO}_{2}$ : pourcentage d'oxygène insufflé; PMax: pression maximale en $\mathrm{cm} \mathrm{H}_{2} \mathrm{O}$; FR: fréquence respiratoire en nombre de coups par minute; Rapport I/E: rapport des durées inspiratoire/cxpiratoire; nb cycles retenus: nombre de cycles passifs analysés.

analyser les cycles en termes de mécanique ventilatoire, seuls ceux où le nouveau-né était totalement passif ont été retenus (Tableau II). Parmi ces cycles, on a pu en sélectionner 11 pour lesquels la phase de plateau à pression maximale était suffisamment longue (cf. Figure 3) pour que le débit se stabilise en fin d'inspiration. Lorsqu'il n'y a pas de fuite au niveau de la sonde, et quand le poumon a atteint un état d'équilibre élastique avec la pression imposée par le respirateur avant la fin de cette phase de plateau, le débit retombe à une valeur nulle. Lorsqu'il y a une fuite autour de la sonde, le débit ne revient pas à zéro mais se stabilise à une valeur qui est celle du débit de fuite, comme l'illustrent les courbes de la Figure 3.
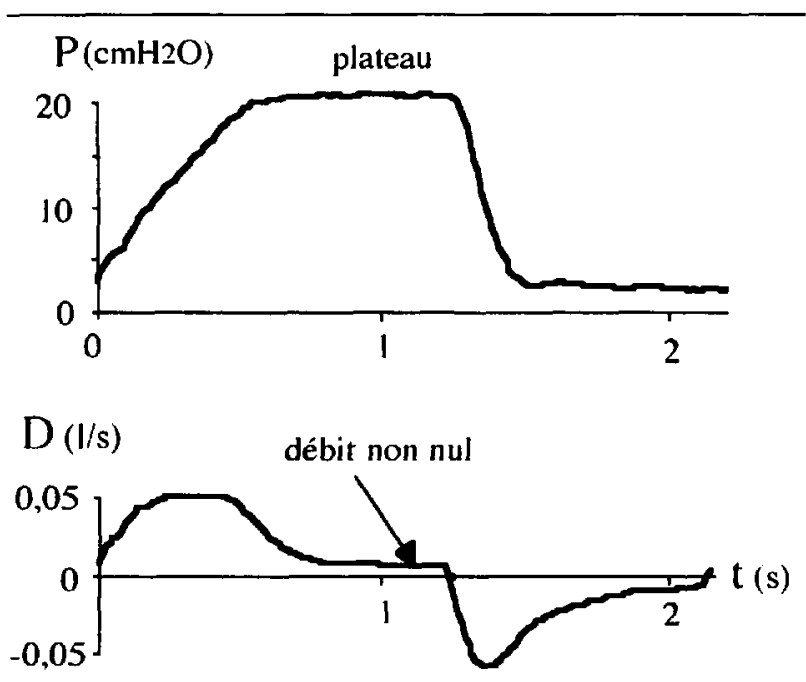

FIGURE 3 Caractérisation graphique de la fuite

Pour ce cycle, l'inspiration occupe les $2 / 3$ du temps total du cycle. La courbe de pression (P) imposée par le respirateur montre un plateau pendant près de la moitié de l'inspiration. Pendant ce plateau, le débit (D) ne revient pas à zéro mais se stabilise à une valeur qui est le débit de fuite.

\section{Traitement des données}

1. SAISIE INFORMATISÉE DES COURBES ET CALCUL DE $R f$

Les signaux $P(t)$ et $D(t)$ sont fournis par les respirateurs (César/TAEMA et Babylog 8000/Draeger) sous forme de tensions électriques variables disponibles sur deux connecteurs situés à l'arrière de l'appareil. Pour les enregistrements, les connecteurs ont été reliés par des câbles à une carte d'acquisition analogique/numérique équipant un micro-ordinateur PC compatible. Un programme, ${ }^{5}$ permet de 1) piloter la carte pour acquérir les signaux à une fréquence d'échantillonnage donnée ( $200 \mathrm{~Hz}$ pour les nouveau-nés et $100 \mathrm{~Hz}$ pour les simulations), 2) sauvegarder les données numérisées sous une forme exploitable par un tableur. Pour chaque cycle retenu ( 11 chez les nouveau-nés et 12 avec le modèle de poumon), nous avons calculé la résistance de fuite ( $R$ f,calc) selon l'équation (6) (donnée en annexe) après correction de la pression selon l'équation (8) avec les coefficients $\mathrm{K}_{1}$ et $\mathrm{K}_{2}$ correspondants.

\section{MESURE DE $R f$ PENDANT la paUse}

Afin d'obtenir une valeur de référence pour la résistance de fuite $(R f$, mes), nous avons réalisé une mesure directe du débit et de la pression de fuite dans les deux situations expérimentales.

Chez le nouveau-né, nous avons mesuré le débit de fuite pendant la pause sur les cycles présentant un plateau de pression suffisamment long pour que le débit se stabilise. La résistance de fuite mesurée $(R f$, mes) est obtenue en divisant, pour chacun des 11 cycles retenus, la moyenne de la pression trachéale (pression mesurée pendant la pause, corrigée de la résistance du tube trachéal) par le débit moyen. Pour les simulations sur modèle de poumon, nous avons d'abord estimé le débit pulmonaire $(D p(t))$ : il est égal à la vitesse de variation du volume du modèle de poumon $(d V(t) / d t)$ qui peut être estimée à partir de la pression dans le soufflet $(P A(t))$ et de l'élastance du soufflet $(E)$ : 


$$
D p(t)=\frac{d V}{d t}=\frac{d\left(P_{A}(t) \mid E\right)}{d t}=\frac{1 d P_{A}(t)}{E d t}
$$

Nous avons vérifié que l'élastance du modèle de poumon est bien linéaire ce qui permet d'estimer le volume alvéolaire et sa vitesse de variation à partir de $P A(t)$. Le débit de fuite est calculé (cf. équation (1)) comme la différence entre le débit mesuré à l'entrée des voies aériennes $(D(t))$ et le débit pulmonaire $(D p(t)) . R f$, mes est alors obtenue pour chaque cycle en calculant la pente de la relation $P A(t)=R f$,mes. $D p(t)$ par régression linéaire. Seules les phases de quasi-stabilité de $P_{A}(t)$ ont été retenues car, en dehors
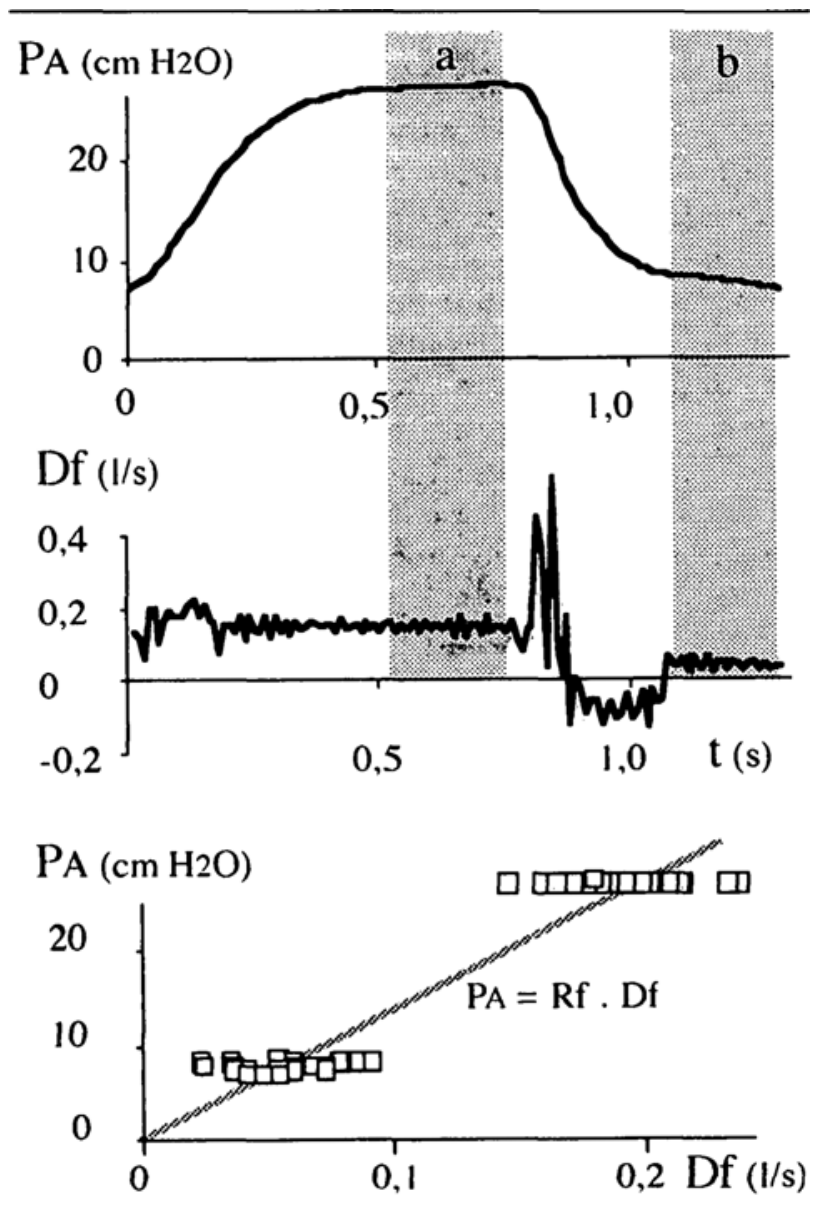

FIGURE 4 Mesure de $R f$ à partir de $P A(t)$ et $D f(t)$

La pression dans le soufflet $(P A(t))$ est assimilée à la pression à l'extrémité de la sonde trachéale et le débit de fuite $(D f(t))$ est obtenu par la formule $D f(t)=D(t)-D p(t)$. (Dp $(t)$ est calculé selon l'équation (9)). Les zones a et $b$ retenues correspondent aux deux pluses à débit de fuite à peu près constant (il y a une PEEP imposée, ce qui explique que le débit de fuite en fin d'expiration n'est pas nul). La résistance de fuite est obtenue comme la pente de la droite de régression du nuage de points, passant par l'origine. de ces phases comme le montre la Figure 4, le débit de fuite calculé était trop variable.

\section{ESTIMATION DE LA PEEP PAR LA MÉTHOdE DES MOINDRES CARRÉS}

Afin d'évaluer l'apport de la correction du débit dans l'estimation des paramètres de la mécanique ventilatoire, nous avons estimé l'élastance (E), la résistance (R) et la PEEP totale (imposée + intrinsèque), à partir des signaux débit et pression obtenus chez les nouveau-nés et sur le modèle de poumon, par la méthode des moindres carrés (Modèle 1 de la référence ${ }^{2}$ ) avant et après correction du débit. Pour les simulations sur modèle de poumon, l'élastance était constante ( $100 \mathrm{~cm} \mathrm{H} \mathrm{H}_{2} \mathrm{O} \cdot \mathrm{L}^{-1}$ ) et la résistance négligeable. Nous avons comparé les estimations de l'élastance et de la PEEP avant et après correction du débit avec les mesures obtenues à partir des courbes de la $P A$ pour l'élastance $(E=$ pente de la relation $P A=f(V))$ et la PEEP (PEEPmes $=P A$ de fin d'expiration). Chez les nouveau-nés, nous avons comparé la PEEP estimée par moindres carrés à la PEEP imposée, seul paramètre accessible sur les 32 cycles enregistrés.

\section{STATISTIQues}

Pour évaluer le niveau de concordance entre les valeurs obtenues par notre méthode et les valeurs mesurées, nous avons utilisé la régression linéaire et nous avons calculé les limites de confiance de la différence (test $\mathrm{t}$ pairé). Les résultats sont exprimés comme la moyenne \pm 2 écarts types. Nous avons aussi tracé le graphique de la différence entre les méthodes en fonction de leur moyenne, comme proposé par Bland et Altman. ${ }^{6}$ Nous estimons qu'une variabilité de la différence (entre les deux méthodes) de $10 \%$ de la valeur moyenne des $R f$ est acceptable pour que les deux méthodes soient interchangeables, sans qu'il y ait un risque de mauvaise interprétation clinique. Les limites d'accord entre les deux méthodes seront déterminées en utilisant les limites de précision et le biais (différence moyenne non nulle). Selon la largeur des intervalles obtenus et la variation des différences, nous pourrons juger du degré d'accord. ${ }^{6}$

\section{Résultats}

\section{Simulations}

L'estimation de l'élastance par la méthode des moindres carrés a été améliorée de $10 \%\left(87 \pm 0,5 \mathrm{~cm} \mathrm{H}_{2} \mathrm{O} \cdot \mathrm{L}^{-1}\right.$ avant correction du débit, $96 \pm 0,5 \mathrm{~cm} \mathrm{H} \mathrm{H}_{2} \mathrm{O} \cdot \mathrm{L}^{-1}$ après correction pour une valeur mesurée de $98 \pm 0,5 \mathrm{~cm}$ $\left.\mathrm{H}_{2} \mathrm{O} \cdot \mathrm{L}^{-1}\right)$. Les résultats de la comparaison entre $R f$,calc et $R f$,mes obtenus pendant les simulations sont présen- 


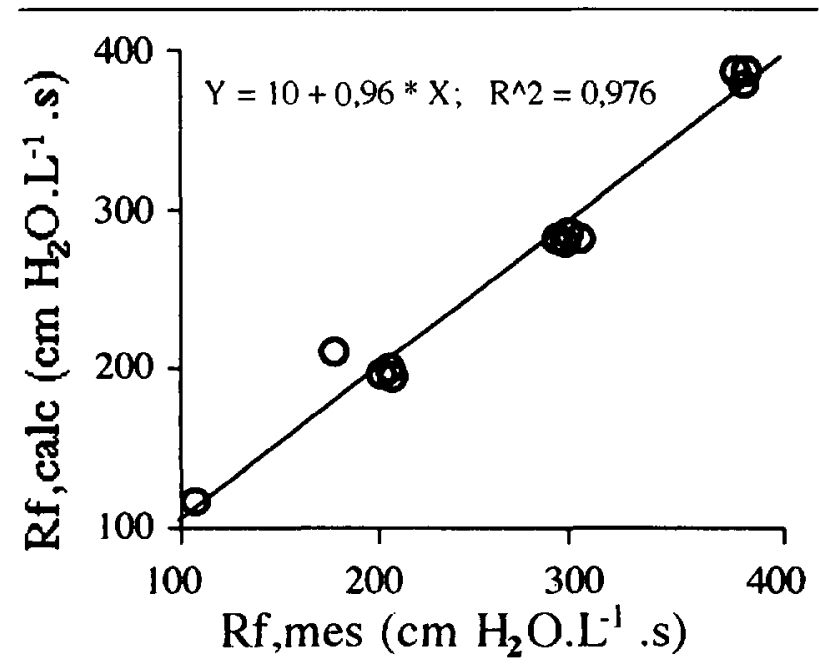

FIGURE 5 Comparaison de $R f$ estimée $(R f, c a l c)$ avec $R f$ mesurée directement ( $R f, m e s)$ au cours des simulations.

La ligne continue représente la droite de régression. La pente n'est pas statistiquement différente de $l$ et l'ordonnée à l'origine n'est pas statistiquement différente de 0 ( $P<0,05$ dans les 2 cas).

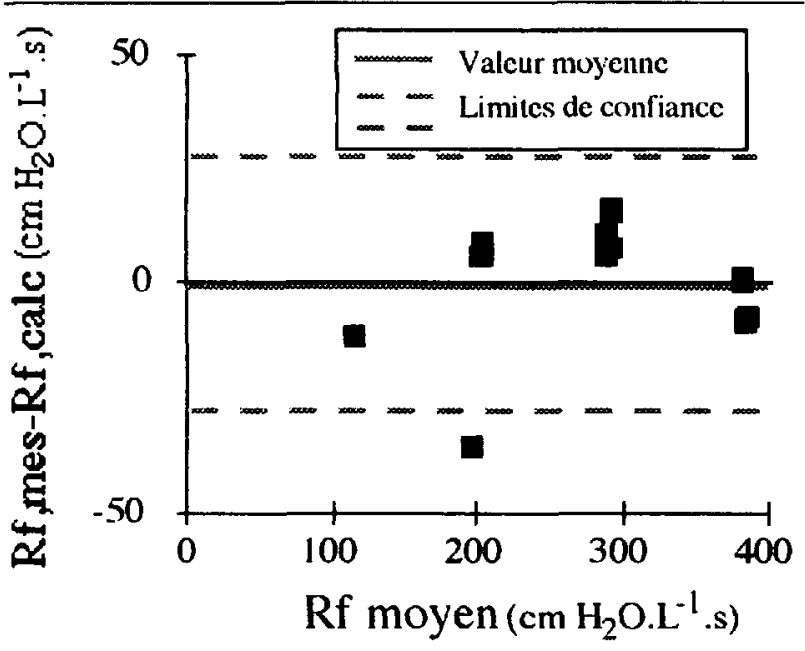

FIGURE 6 Niveau de concordance entre $R f$,calc et $R f$,mes pour les simulations

L'amplitude de la zone de confiance à $95 \%$ est inférieure à $20 \%$ de la valeur moyenne de $R f$.

tés sur les Figures 5 et 6 . La différence moyenne entre $R f$,calc et $R f$,mes n'est pas statistiquement différente de $0\left(-0,8 \pm 13,8 \mathrm{~cm} \mathrm{H} \mathrm{H}_{2} \mathrm{O} \cdot \mathrm{L}^{-1} \cdot \mathrm{sec}^{-1}\right)$. Les écarts entre $R f$,calc et $R f$,mes se situent, à l'exception d'un point, dans un zone d'amplitude inférieure à $20 \%$ de la valeur moyenne de $R f$. L'intervalle de confiance à $95 \%$ pour le biais va de $-9,7$ à $8,0 \mathrm{~cm} \mathrm{H} \mathrm{H}_{2} \mathrm{O} \cdot \mathrm{L}^{-1} \cdot \mathrm{sec}^{-1}$, pour la limite inférieure d'accord de $-43,8$ à $-13,0 \mathrm{~cm} \mathrm{H} \mathrm{H}_{2} \mathrm{O} \cdot \mathrm{L}^{-1} \cdot \mathrm{sec}^{-1}$ et pour la limite supérieure d'accord de 11,4 à $42,1 \mathrm{~cm}$ $\mathrm{H}_{2} \mathrm{O} \cdot \mathrm{L}^{-1} \cdot \mathrm{sec}^{-1}$. Ces intervalles sont relativement étroits

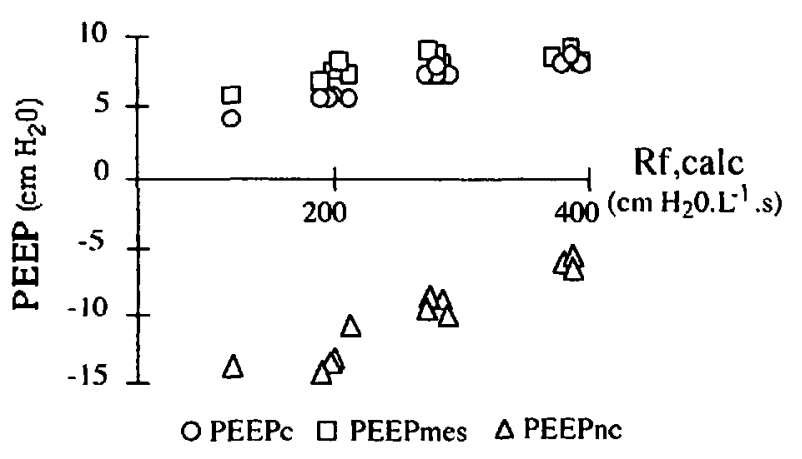

FIGURE 7 Effet de la correction du débit sur l'estimation de la PEEP par la méthode des moindres carrés

PEEPnc: PEEP estiméc avant correction du débit; PEEPc: PEEP estimée après correction du débit; PEEPmes: valcur de la pression dans le soufflet à la fin de l'expiration.

par rapport à la valeur moyenne de $R f(267,4 \mathrm{~cm}$ $\left.\mathrm{H}_{2} \mathrm{O} \cdot \mathrm{L}^{-1} \cdot \mathrm{sec}^{-1}\right)$, reflétant la faible variation des différences et donc la possibilité d'interchanger les deux méthodes.

La PEEP estimée par la méthode des moindres carrés passe en moyenne de $-9,9 \pm 3,1 \mathrm{~cm} \mathrm{H}_{2} \mathrm{O}$, avant correction, à $6,7 \pm 1,3 \mathrm{~cm} \mathrm{H} \mathrm{H}_{2} \mathrm{O}$, après correction. Cependant cette valeur est supérieure $(P<0,05)$ à la PEEP mesurée moyenne $\left(7,8 \pm 0,8 \mathrm{~cm} \mathrm{H}_{2} \mathrm{O}\right)$. L'écart moyen entre PEEP estimée par les moindres carrés après correction du débit et PEEP mesurée est faible $(1,2 \pm 0,6 \mathrm{~cm}$ $\mathrm{H}_{2} \mathrm{O}$ ). On voit sur la Figure 7 que, comme pour les nouveau-nés, l'erreur sur l'estimation de la PEEP avant correction augmente lorsque $R f$ diminue, c'est à dire lorsque le débit de fuite augmente. Avant correction la PEEP estimée était négative alors qu'après correction elle se rapproche notablement (erreur inférieure à $3 \mathrm{~cm}$ $\mathrm{H}_{2} \mathrm{O}$ ) de la valeur mesurée directement.

\section{NOUVEAU-NÉS}

Pour tous les cycles retenus (les 11 présentant un plateau assez long pour que le débit se stabilise en fin d'inspiration) le débit corrigé est nul en fin d'inspiration, comme illustré sur la Figure 8.

Les résultats de la comparaison entre $R f$,calc et $R f$, mes chez les nouveau-nés sont présentés sur les Figures 9 et 10. La différence moyenne entre $R f$, calc et $R f$,mes n'est pas différente de $0(87 \pm 99 \mathrm{~cm}$ $\left.\mathrm{H}_{2} \mathrm{O} \cdot \mathrm{L}^{-1} \cdot \sec ^{-1}\right)$. La méthode de calcul de $R f$ est en accord avec la mesure directe comme le montre la relative étroitesse de la zone de confiance à 95\% par rapport aux valeurs moyennes de $R f$. En effet, l'intervalle de confiance à $95 \%$ pour le biais va de 20,4 à $153,5 \mathrm{~cm}$ $\mathrm{H}_{2} \mathrm{O} \cdot \mathrm{L}^{-1} \cdot \mathrm{sec}^{-1}$, pour la limite inférieure d'accord de $-226,5$ à $4,1 \mathrm{~cm} \mathrm{H}_{2} \mathrm{O} \cdot \mathrm{L}^{-1} \cdot \mathrm{sec}^{-1}$ et pour la limite supérieure d'accord de 169,8 à $400,3 \mathrm{~cm} \mathrm{H} \mathrm{H}_{2} \mathrm{O} \cdot \mathrm{L}^{-1} \cdot \mathrm{sec}^{-1}$. 


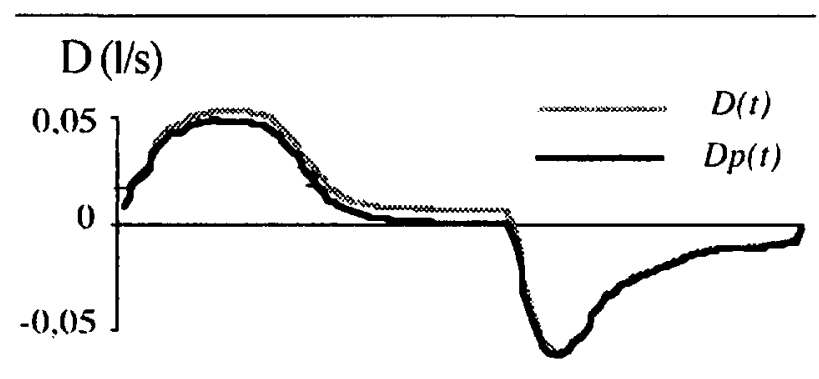

FIGURE 8 Exemple de débit corrigé après calcul de $R f$ L'efficacité de la correction de la fuite peut être mise en évidence par la valeur nulle du débit corrigé $(D p(t))$ pendant le plateau de pression de fin d'inspiration.

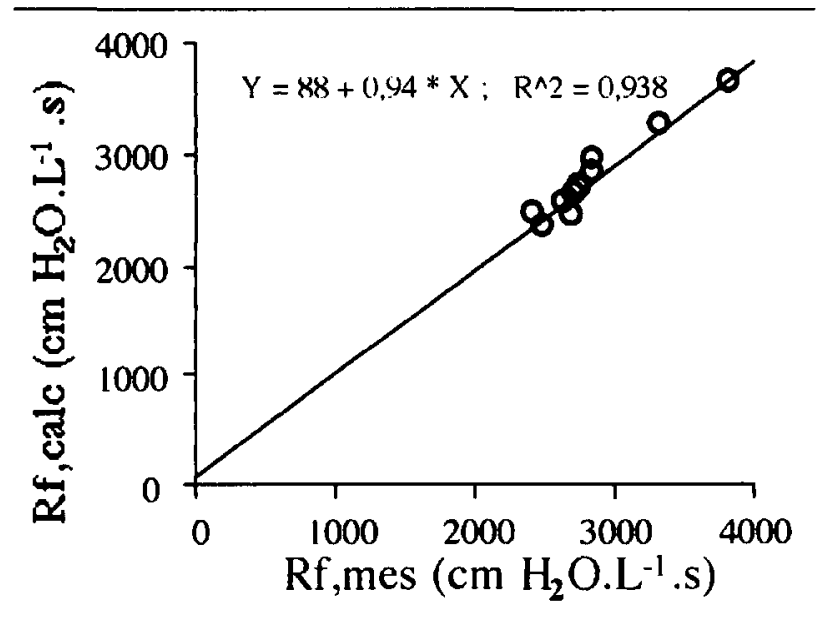

FIGURE 9 Comparaison de $R f$ estimée ( $R f$,calc) avec $R f$

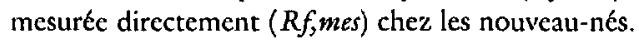

La ligne continue représente la droite de régression. La pente n'est pas statistiquement différente de 1 et l'ordonnée à l'origine n'est pas statistiquement différente de $0(P<0,05$ dans les 2 cas).

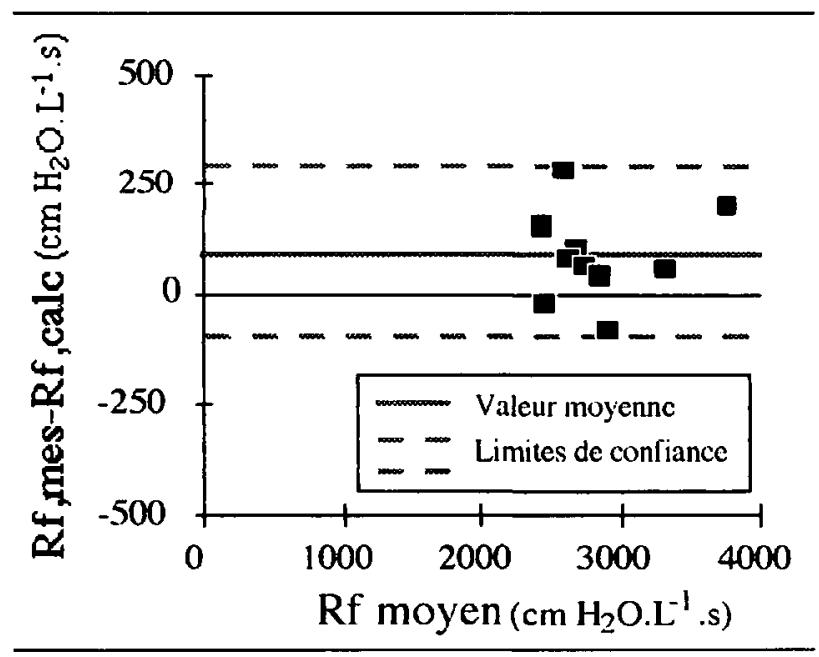

FIGURE 10 Niveau de concordance entre $R f$,calc et $R f$,mes chez les nouveau-nés

L'amplitude de la zone de confiance à $95 \%$ est inférieure à $20 \%$ de la valeur moyenne de $R f$.

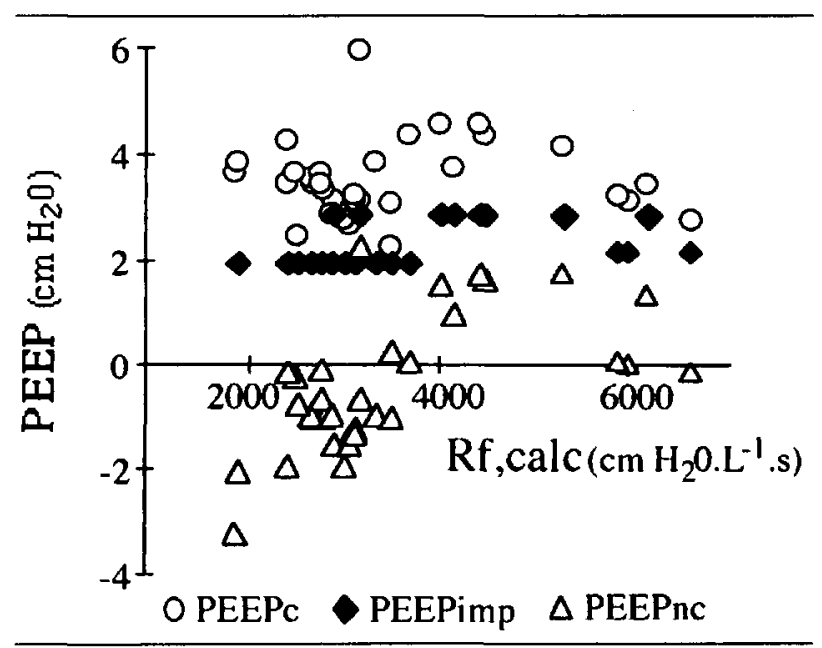

FIGURE 11 Effet de la correction du débit sur l'estimation de la PEEP par la méthode des moindres carrés

PEEPnc: PEEP estimée avant correction du débit; PEEPc: PEEP estimée après correction du débit; PEEPimp: PEEP imposée par le ventilateur.

Ces intervalles sont relativement petits par rapport à la valeur moyenne de $R f\left(2828, \mathrm{I} \mathrm{cm} \mathrm{H} \mathrm{O}_{2} \mathrm{O} \cdot \mathrm{L}^{-1} \cdot \mathrm{sec}^{-1}\right)$, illustrant donc un lien étroit entre les deux méthodes. La correction du débit améliore nettement l'estimation de la PEEP totale par la méthode des moindres carrés puisque la PEEP estimée passe en moyenne de $-0,3 \pm$ $3,6 \mathrm{~cm} \mathrm{H} \mathrm{H}_{2} \mathrm{O}$, avant correction, à $1,3 \pm 0,7 \mathrm{~cm} \mathrm{H}_{2} \mathrm{O}$, après correction. Cependant cette valeur est significativement supérieure $(P<0,05)$ à la PEEP imposée moyenne $\left(2,2 \pm 0,4 \mathrm{~cm} \mathrm{H}_{2} \mathrm{O}\right)$. Sur la Figure 11, les valeurs de PEEP estimées ou imposées sont portées en fonction de la valeur calculée de $R f$. L'erreur sur l'estimation de la PEEP avant correction augmente lorsque $R f$ dininue, c'est à dire lorsque le débit de fuite augmente.

\section{Discussion}

Nous avons montré qu'il est possible de corriger le débit instantané par une estimation simple de la résistance de fuite $R f$. L'intérêt de cette méthode repose essentiellement sur le fait que près du tiers des nouveau-nés présente une fuite. ${ }^{7}$ On peut espérer retirer un bénéfice clinique de l'application de cette nouvelle méthode à deux niveaux: le premier sera de guider le clinicien dans le choix de la sonde à utiliser, le second sera d'améliorer la surveillance de la mécanique ventilatoire: en effet, la mauvaise mesure du débit liée à l'existence d'une fuite entraîne une mauvaise estimation de la mécanique ventilatoire. L'hypothèse que $R f$ ne dépend pas du débit de fuite est à la base de notre méthode. Les mesures exposées à la Figure 4 ne permettent en aucun cas de valider ou d'infirmer cette hypothèse. Nous estimons cependant que les faibles 
débits de fuite habituellement rencontrés (sur la Figure 8, on peut estimer ce débit à environ $10 \mathrm{ml} \cdot \mathrm{sec}^{-1}$ ) permettent de supposer l'hypothèse vérifiée dans ces conditions. Dans le cas d'enfants normaux, on peut considérer que les variations du diamètre de la trachée sur un cycle respiratoire sont suffisamment petites pour que l'on puisse admettre l'hypothèse que le rapport entre le diamètre de la trachée et le diamètre du tube endotrachéal est constant. Ceci n'est cependant pas vrai dans le cas d'une trachéomalacie, caractérisé par un ramollissement de la trachée causée par une dégénérescence des cartilages. ${ }^{8}$ Notre méthode repose aussi sur l'estimation de la pression à l'extrémité de la sonde à partir du signal de pression donné par le ventilateur et des caractéristiques résistives de la sonde. Ces dernières ne peuvent être connues parfaitement pour deux raisons: 1 ) les lois d'écoulement font que la résistance n'est pas la même à l'inspiration et à l'expiration ${ }^{4}$ et 2) en situation clinique, on sait que la sonde va s'encombrer progressivement. Cet encombrement est levé périodiquement par des aspirations et il pourrait être estimé par des méthodes directes de mesure de la surface de section du conduit. $^{9}$

En ce qui concerne les simulations, nous n'avons pas essayé de simuler plusieurs valeurs des paramètres mécaniques car ce n'était pas le but du travail. L'important était de pouvoir estimer directement le débit de fuite pour différentes valeurs de $R f$. Dans nos simulations, la résistance de fuite était dix fois plus faible que chez les nouveau-nés, ce qui aurait pu entraîner une plus forte non linéarité de la fuite. L'ensemble de nos mesures semble montrer qu'il n'en était rien. Les résistances de fuite obtenues en simulation sont environ dix fois inférieures à celles obtenues en clinique. Ces valeurs sont cohérentes avec le fait que la sonde trachéale de diamètre $5,5 \mathrm{~mm}$ (en simulation) est dix fois moins résistive que celle utilisée avec les nouveau-nés.

En ce qui concerne les enregistrements sur les nouveau-nés, il a été relativement difficile de trouver des cycles présentant les caractéristiques requises, c'est à dire avec des patients complètement passifs, présentant une fuite et un plateau inspiratoire long. Nous avons donc peu de valeurs de contrôle puisque nous avions choisi, pour de simples raisons d'éthique, de ne modifier en aucun cas les paramètres ventilatoires fixés par le médecin traitant. La mesure de la résistance de fuite n'a été réalisable que lorsque la pause durait suffisamment longtemps pour que le débit de fin d'inspiration atteigne une valeur stable. Le débit de fuite a alors pu être estimé par le rapport entre la pression maximale et la résistance de fuite, donnant des valeurs comprises entre 4 et 7,5 $\mathrm{ml} \cdot \mathrm{sec}^{-1}$ pour des débits de l'ordre de $100 \mathrm{ml} \cdot \mathrm{sec}^{-1}$ Dans tous les autres cas, la résistance de fuite estimée par le rapport Pression de fin d'inspiration/débit de fin d'inspiration est très nettement inférieur à $R f$ calculée par notre méthode ce qui laisse penser que le débit mesuré n'est pas uniquement le débit de fuite mais que le poumon continue à se gonfler. La méthode proposée de calcul des résistances de fuite présente l'avantage que $R f$ est estimée pour chaque cycle sur lequel elle est appliquée. Il est donc possible de corriger le débit chaque fois qu'il est mesuré et de mettre en oeuvre les méthodes d'estimation des paramètres de mécaniques ventilatoires avec un débit corrigé même si la fuite change entre deux estimations. En pratique clinique, dans le cas de la présence de fuites trop importantes et d'une hypoventilation alvéolaire, l'action du clinicien sera de changer le diamètre du tube trachéal plutôt que de prendre le risque d'une surcompensation ou d'un barotraumatisme pulmonaire. Par ailleurs, il est évident que notre méthode ne pourra pas être utilisée avec des nouveau-nés très peu passifs.

L'application numérique et graphique de l'étude statistique de Bland et Altman ${ }^{6}$ a mis en évidence un lien étroit entre les deux méthodes proposées. Il est vrai cependant, que la répétition des mesures obtenues chez un même sujet (le sujet 2) diminue la variabilité interindividus. Nous n'avons utilisé la méthode des moindres carrés que pour évaluer l'apport de la correction du débit en cas de fuite. Nous avons choisi cette méthode car elle permet d'estimer la PEEP totale (imposée + intrinsèque), importante en clinique, à l'inverse de la FFT ${ }^{10}$ mais le modèle retenu aurait pu être nettement amélioré. Le fait que la résistance "des voies aériennes" est négligeable dans notre simulation n'allait pas dans le sens de la complexification du modèle à appliquer. ${ }^{2,11}$ Il semble normal que la PEEP estimée soit supérieure en moyenne à la PEEP imposée car dans certains cas (comme celui illustré sur la Figure 11) il y a probablement de la PEEP intrinsèque qui est prise en compte par la méthode des moindres carrés.

En conclusion, nous pensons que cette méthode simple à mettre en oeuvre dès que l'on connaît les caractéristiques du tube trachéal, devrait être appliquée avec succès à la surveillance des caractéristiques de mécanique ventilatoire des nouveau-nés et pourrait être utilisée avec profit chez les adultes de façon systématique puisque, en l'absence de fuite ( $R f$ infinie), la correction du débit n'a pas lieu.

\section{Remerciements}

L'étude s'est déroulée au sein et avec le soutien du Groupe de Recherche et Modélisation en Mécanique Ventilatoire (Grenoble-Lyon). La société Dräger a 
gracieusement prêté une carte interface permettant de connecter le ventilateur Babylog 8000 à notre système d'acquisition. PY Carry a été soutenu par les Hospices Civils de Lyon. Le travail a été soutenu par la Délégation Régionale à la Recherche Clinique du CHU de Grenoble.

\section{Références}

1 Pérez Fontán J], Heldt GP, Gregory GA. The effect of a gas leak around the endotracheal tube on the mean tracheal pressure during mechanical ventilation. Am Rev Respir Dis 1985; 132: 339-42.

2 Peslin R, Felicio da Silva J, Chabot F, Duvivier C. Respiratory mechanics studied by multiple linear regression in unsedated ventilated patients. Eur Respir J 1992; 5: 871-8.

3 Ubl RR, Lewis FJ. Digital computer calculation of human pulmonary mechanics using a least squares fit technique. Computers and Biomedical Research 1974; 7: 489-95.

4 Chang HK, Mortola JP. Fluid dynamic factors in tracheal pressure measurement. J Appl Physiol 1981; 51: 218-25.

5 Baconnier PF, Carry P- $\Upsilon$, Eberbard A, Perdrix J-P, Fargnoli J-M. A computer program for automatic measurement of respiratory mechanics in artificially ventilated patients. Comput Methods Programs Biomed 1995; 47: 205-20.

6 Bland JM, Altman DG. Statistical methods for assessing agreement between two methods of clinical measurement. Lancet 1986; 8: 307-10.

7 Seear $M$, Wensley $D$, Werner $H$. Comparison of three methods for measuring respiratory mechanics in ventilated children. Pediatr Pulmonol 1991; 10: 291-5.

8 Wittenborg $M H$, Gyepes MT, Crocker D. Tracheal dynamics in infants with respiratory distress, stridor, and collapsing trachea. Radiology 1967; 88: 653-62.

9 Van Surell C, Louis B, Lofaso F, et al. Acoustic method to estimate the longitudinal area profile of endotracheal tubes. Am J Respir Crit Care Med 1994; 149: 28-33.

10 Peslin R, Gallina C, Saunier C, Duvivier C. Fourier analysis versus multiple linear regression to analyse pressure-flow data during artificial ventilation. Eur Respir J 1994; 7: 2241-5.

11 Kano S, Lanteri CJ, Duncan AW, Sly PD. Influence of nonlinearities on estimates of respiratory mechanics using multilinear regression analysis. J Appl Physiol 1994; 77: 1185-97.

Annexe: Calculs détaillés de la résistance de fuite Ecoulement à l'extrémité de la sonde:

A tout instant, le débit dans la sonde trachéale est la somme du débit allant vers le poumon et de celui passant par la fuite:

$$
D=D f+D p
$$

\section{Ecoulement dans la fuite:}

Si on suppose que la résistance de fuite $(R f)$ ne dépend pas du débit ou de la pression, on peut écrire l'équation qui caractérise cet écoulement:

$$
P \operatorname{tr}=D f . R f
$$

Les intégrales sur une durée données de 2 quantités égales étant égales, on peut écrire:

$$
\int P t r . d t=\int R f . D f . d t=R f . \int D f . d t
$$

Or on peut raisonnablement supposer qu'en état stationnaire (pas de variation du volume courant pulmonaire ni du volume de fin d'expiration d'un cycle à l'autre) l'intégrale sur un cycle du débit des voies aériennes est nulle, qu'il y ait ou non hyperinflation dynamique.

$$
\int_{\text {crde }} D p \cdot d t=0
$$

Si l'on calcule l'intégrale sur un cycle du débit dans la sonde trachéale, on obtient, en utilisant successivement (1) et (4):

$$
\int_{\text {octl }} D \cdot d t=\int_{\text {cyde }}(D p+D f) \cdot d t=\int_{\text {cycte }} D p \cdot d t+\int_{c y d t} D f \cdot d t=\int_{c y c l e} D f \cdot d t
$$

A l'aide des équations (3) et (5) on démontre alors aisément que :

$$
R f=\frac{\int_{c y c t c} P t r . d t}{\int_{c y c l e} D \cdot d t}
$$

Il est alors possible de calculer le débit pulmonaire à chaque instant :

$$
D p(t)=D-D f=D(t)-\frac{P \operatorname{tr}(t)}{R f}
$$

